\title{
Contact problem on indentation of an elastic half-plane with functionally-graded coating in presence of tangential stresses on the surface
}

\author{
Sergei S. Volkov", Andrey S. Vasiliev, and Evgeniy V. Sadyrin \\ Research and Education Center "Materials", Don State Technical University, 344000 Rostov-on-Don, \\ Russia
}

\begin{abstract}
Plane contact problem on indentation of an elastic half-plane with functionally graded coating by a parabolic punch is considered. The surface of the half-plane is additionally subjected to distributed tangential stresses in a certain region different from contact area. The contact area is assumed to be asymmetric with respect to the center of the punch. Tangential stresses are represented in the form of Fourier series. The problem is reduced to the solution of two dual integral equations over even and odd functions describing distribution of normal contact stresses. The bilateral asymptotic method is used to solve these equations. Approximated analytical solutions asymptotically exact for both the small and large values of relative coating thickness are constructed.
\end{abstract}

\section{Introduction}

Nowadays layered and functionally graded coatings for the protection of structural elements from wear, oxidation, cracking, as well as mechanical, temperature and other types of influences are widely used [1-4]. Recently the effectiveness of functionally graded materials in heavily loaded point elastohydrodynamically lubricated contacts with straight lubricant entrainment was studied [5-9]. The problems were studied based on the method of matched asymptotic expansions which allows one to split the problem into two separate problems: a dry contact problem for functionally graded elastic materials and an elastohydrodynamically lubricated problem for functionally graded materials. In these problems the tangential stresses on the surface were assumed to be absent, however, it was also shown [10] that the presence of tangential stresses caused by friction between a lubricant and surface of the coating can be of fundamental importance for the elastohydrodynamically lubricated contact. At the same time, during problem of dry contact statement, it was not taken into account that in the hydrodynamic contact the area of contact between the indenter and the coating is asymmetric relative to the center of the indenter.

In the present paper we consider the contact problem on the dry contact of a parabolic indenter with a functionally graded coating on a homogeneous half-plane, taking into account the distribution of tangential stresses on the surface of the coating. The solution of

\footnotetext{
*Corresponding author: fenix_rsu@mail.ru
} 
the problem is constructed in a semi-analytical form using a bilateral asymptotic method. It was taken into account that the contact zone of the indenter with the coating is asymmetric relative to the center of the indenter, as well as the region in which tangential stresses are given. These results provide a foundation for the solution of elastohydrodynamically lubricated contact problems in the presence of tangential stresses on the coating surface.

\section{Problem statement}

A rigid parabolic punch of radius $R$ interacts with the surface $(z=0)$ of an elastic inhomogeneous half-plane. The half-plane consists of a functionally graded coating of thickness $H$, Young's modulus $E_{c}(\mathrm{z})$ and Poisson's ratio $v_{c}(z)$, and a homogeneous substrate with Young's modulus $E_{s}$ and Poisson's ratio $v_{s}$. The coating and the substrate are glued without sliding. Under the action of normal centrally applied force $P$ the punch moves at a distance $\delta$ downward the $z$-axis. Outside of the contact area the surface is traction-free. Tangential stresses are acting on the region $[-d, c]$, and their values are assumed to be known. Therefore, the boundary conditions take the form:

$$
z=0:\left\{\begin{array} { c } 
{ \sigma _ { z } ^ { ( c ) } = 0 , \quad x < - b , \quad x > a } \\
{ w ^ { ( c ) } = - \delta + \frac { x ^ { 2 } } { 2 R } , \quad - b \leq x \leq a }
\end{array} \quad \left\{\begin{array}{ll}
\tau_{x z}^{(c)}=\tau_{0}(x), & -d \leq x \leq c \\
\tau_{x z}^{(c)}=0, & x>c, \quad x<-d
\end{array}\right.\right.
$$

It is required to determine the contact normal stresses under the punch:

$$
\left.\sigma_{z}\right|_{z=0}=-p_{0}(x),-b \leq x \leq a, p_{0}(a)=0, p_{0}(-b)=0
$$

Further, without loss of generality, we assume that $d-b=c-a, a>b$.

\section{Problem solution}

Using the technique of integral Fourier transforms of the following form

$$
f(x, z)=\frac{1}{2 \pi} \int_{-\infty}^{\infty} \bar{f}(\alpha, z) e^{-i \alpha x} d \alpha, \quad \bar{f}(\alpha, z)=\int_{-\infty}^{\infty} f(x, z) e^{i \alpha x} d x
$$

and the following change of variables

$$
\begin{gathered}
x=\frac{a-b}{2}+\frac{b+a}{2} x^{\prime}, \alpha^{\prime}=\frac{a+b}{2} \alpha, \lambda=\frac{H}{a}, l=\frac{2 c-a+b}{b+a}, L_{i j}^{\prime}(\alpha)=L_{i j}\left(\frac{\alpha}{H}\right) \\
\left\{p_{0}^{\prime}, \tau_{0}^{\prime}\right\}\left(x^{\prime}\right)=\left\{p_{0}, \tau_{0}\right\}\left(\frac{a-b}{2}+\frac{b+a}{2} x^{\prime}\right), \quad\left\{\bar{p}_{0}, \bar{\tau}_{0}\right\}(\alpha)=e^{i \alpha^{\prime}\left(\frac{a-b}{a+b}\right)} \frac{b+a}{2}\left\{\bar{p}_{0}^{\prime}, \bar{\tau}_{0}^{\prime}\right\}\left(\alpha^{\prime}\right)
\end{gathered}
$$

and omitting the "prime" sign, the stated problem reduces to solution of the following system of integral equations 


$$
\begin{aligned}
& \int_{-\infty}^{\infty}\left(\frac{i L_{13}(\lambda \alpha)}{E_{13}^{\prime}(0)} \bar{\tau}_{0}(\alpha)+\frac{L_{33}(\lambda \alpha)}{E_{33}^{\prime}(0)} \bar{p}_{0}(\alpha)\right) \frac{e^{-i \alpha x} d \alpha}{|\alpha|}=\frac{2 \pi}{b+a}\left(\delta-\frac{1}{2 R}\left(\frac{a-b}{2}+\frac{b+a}{2} x\right)^{2}\right),|x| \leq 1 \\
& \int_{-\infty}^{\infty} \bar{p}_{0}(\alpha) e^{-i \alpha x} d \alpha=0, \quad|x|>1, \quad \int_{-\infty}^{\infty} \bar{\tau}_{0}(\alpha) e^{-i \alpha x} d \alpha=0, \quad|x|>l
\end{aligned}
$$

In the equation (4) $L_{i j}(\alpha)$ are compliance functions of an inhomogeneous half-space. Their values, in the case of a sufficiently arbitrary law of elastic moduli variation by the coating thickness, can be obtained numerically using the approach proposed in [11]. Functions $L_{i j}(\alpha)$ possess the following properties: $L_{13}(\alpha)$ is an odd function, $L_{33}(\alpha)$ is an even function. $E_{i j}^{\prime}(z)$ are effective elastic moduli:

$$
E_{33}^{\prime}(z)=\frac{E_{c}(z)}{1-\left(v_{c}(z)\right)^{2}}, \quad E_{13}^{\prime}=\frac{2 E_{c}(z)}{\left(1+v_{c}(z)\right)\left(1-2 v_{c}(z)\right)} .
$$

Let us represent the function $p_{0}(x)$ as a sum of even and odd functions:

$$
p_{0}(x)=p_{+}(x)+p_{-}(x), \bar{p}_{0}(\alpha)=\bar{p}_{+}(\alpha)+i \bar{p}_{-}(\alpha)
$$

Let us also assume that the function $\bar{\tau}_{0}(\alpha)$ can be represented in the form of a segment of a Fourier series on the interval $\left[-l_{c}, l_{c}\right]$ of the following form

$$
\tau_{0}(x)=\sum_{k=0}^{M}\left(a_{k} \cos \left(\tilde{l}_{k} x\right)+b_{k} \sin \left(\tilde{l}_{k} x\right)\right), \tilde{l}_{k}=\pi k l^{-1}
$$

and the compliance function $L_{i j}(\alpha)$ is approximated by the following expression $[11,12]$

$$
L_{i j}(\alpha) \approx \Pi_{i j}(\alpha)=P_{1}^{i j}\left(\alpha^{2}\right) / P_{2}^{i j}\left(\alpha^{2}\right) P_{\{1,2\}}^{i j}\left(\alpha^{2}\right)=\prod_{n=1}^{N_{i j}}\left(\alpha^{2}+\{A, B\}_{i j n}^{2}\right), A_{i j n} \neq B_{l m s}
$$

Then the problem posed can be reduced to solving the following two dual integral equations

$$
\begin{aligned}
& \int_{-\infty}^{\infty} \Pi_{33}(\alpha \lambda) \frac{\bar{p}_{-}(\alpha)}{|\alpha|} \sin (\alpha x) d \alpha=E_{33}^{\prime}(0)\left(I_{-}(x)-\frac{\pi(a-b)}{2 R} x\right),|x| \leq 1 \\
& \int_{-\infty}^{\infty} \bar{p}_{-}(\alpha) \sin (\alpha x) d \alpha=0, \quad|x|>1 \\
& \int_{-\infty}^{\infty} \Pi_{33}(\alpha \lambda) \frac{\bar{p}_{+}(\alpha)}{|\alpha|} \cos (\alpha x) d \alpha= \\
& =E_{33}^{\prime}(0)\left(I_{+}(x)+\frac{2 \pi}{b+a}\left(C-\frac{1}{8 R}\left((a-b)^{2}+(b+a)^{2} x^{2}\right)\right)\right), \quad|x| \leq 1 \\
& \int_{-\infty}^{\infty} \bar{p}_{+}(\alpha) \cos (\alpha x) d \alpha=0, \quad|x|>1
\end{aligned}
$$


In (8) the function form $I_{-}(x)$ is determined by the separation of the odd part. It is performed by calculation of the integral in (4) containing $\bar{\tau}_{0}(\alpha) . I_{+}(x)$ in (9) is determined by the separation of the even part, respectively.

Using the methods and approaches of the works [5, 6, 11-13], analytical solution of the equations (8), (9) can be constructed

$$
p_{0}(x)=\mathrm{X}_{-}(x, \lambda)-\mathrm{X}_{+}(x, \lambda)+\frac{E_{33}^{\prime}(0)(b+a)}{4 R \Pi_{33}(0)} \sqrt{1-x^{2}}
$$

The boundary of the contact zone is determined from equations

$$
2 P+\pi(b+a) \mathrm{Y}_{+}(\lambda)-\pi \frac{E_{33}^{\prime}(0)(b+a)^{2}}{8 R \Pi_{33}(0)}=0, \quad \mathrm{Y}_{-}(\lambda)-\frac{E_{33}^{\prime}(0)(a-b)}{4 R \Pi_{33}(0)}=0
$$

In (10), (11) the following notations are used

$$
\begin{aligned}
& \Phi_{\{-,+\}}(x, A)=A \int_{x}^{1} \frac{t\{s h, \operatorname{ch}\}(A(t-x)) I_{0}(A)-I_{1}(A)\{c h, s h\}(A(t-x))}{\sqrt{1-t^{2}}} d t \\
& \mathrm{Y}_{\{-,+\}}(\lambda)=\frac{E_{33}^{\prime}(0)}{2 \pi E_{13}^{\prime}(0)}\left(\sum_{m=1}^{N_{33}}\{D, C\}_{m} I_{\{0,1\}}\left(A_{33 m} \lambda^{-1}\right)+\sum_{n=1}^{N_{13}} W_{n}^{\{-,+\}} I_{\{0,1\}}\left(B_{13 n} \lambda^{-1}\right)+\right. \\
& \left.+\sum_{k=0}^{M} Z_{k}^{\{-,+\}} I_{\{0,1\}}\left(i \tilde{l}_{k}\right)\right) \\
& \mathrm{X}_{\{-,+\}}(x, \lambda)=\frac{E_{33}^{\prime}(0)}{2 \pi E_{13}^{\prime}(0)}\left(\sum_{m=1}^{N_{33}}\{D, C\}_{m} \Phi_{\{-,+\}}\left(x, A_{33 m} \lambda^{-1}\right)+\sum_{n=1}^{N_{13}} W_{n}^{\{-,+\}} \Phi_{\{-,+\}}\left(x, B_{13 n} \lambda^{-1}\right)+\right. \\
& \left.+\sum_{k=0}^{M} Z_{k}^{\{-,+\}} \Phi_{\{-,+\}}\left(x, \tilde{i}_{k}\right)\right) \\
& W_{n}^{-}=\frac{2 \pi \Pi_{13 n}\left(i B_{13 n}\right)}{\Pi_{33}\left(i B_{13 n}\right)} \sum_{k=0}^{M} a_{k} \frac{(-1)^{k} e^{-l B_{13 n} \lambda^{-1}}}{\widetilde{l}_{k}^{2}+B_{13 n}^{2} \lambda^{-2}}, Z_{k}^{-}=-\frac{2 \pi a_{k} \Pi_{13}\left(\tilde{l}_{k} \lambda\right)}{\Pi_{33}\left(\widetilde{l}_{k} \lambda\right)} \\
& W_{n}^{+}=\frac{2 \pi \lambda \Pi_{13 n}\left(i B_{13 n} \lambda^{-1}\right) e^{-l B_{13 n} \lambda^{-1}}}{\Pi_{33}\left(i B_{13 n}\right) B_{13}} \sum_{k=0}^{\infty} \frac{b_{k} \tilde{l}_{k}(-1)^{k}}{\left(\widetilde{l}_{k}^{2}+B_{13 n}^{2} \lambda^{-2}\right)}, Z_{k}^{+}=2 \pi \frac{b_{k} \Pi_{13}\left(\tilde{l}_{k} \lambda\right)}{\Pi_{33}\left(\widetilde{l}_{k} \lambda\right)}, \\
& \Pi_{i j n}(\alpha)=\frac{\left(A_{i j n}^{2}-B_{i j n}^{2}\right)}{\lambda^{2}} \prod_{m=1, m \neq n}^{N_{i j}} \frac{\alpha^{2}+A_{i j m}^{2}}{\alpha^{2}+B_{i j m}^{2}} .
\end{aligned}
$$

Here $\{D, C\}_{m}$ are unknown constants, determined from the systems of linear algebraic equations obtained by substituting (10) into the initial equation (4), after a series of cumbersome transformations. 


\section{Conclusion}

Due to the application of the bilateral asymptotic method to the solution of the dual integral equations (8), (9), the obtained solution (10) is effective in a wide range of geometrical and physical parameters of the problem (the thickness of the coating, the values of the elastic moduli of the coating and the substrate, the law of variation of the elastic moduli in the coating). The results presented in the paper are of interest for tribology. In particular, using an analogous approach, an iterative algorithm can be implemented to solve the contact problem taking frictional forces into account. Using the method of matched asymptotic expansions an elastohydrodynamically lubricated contact problem [5-9] can be solved taking into account the presence of the tangential stresses on the surface. Also, the results can be used to solve contact problems on the contact of two elastic inhomogeneous bodies, bodies with transversely isotropic and piezoelectric coatings, thermoelastic problems, and so on.

Authors acknowledge the support of the Ministry of Education and Science of Russia in the framework of Governmental Assignment no. 9.1481.2017/4.6. S.S. Volkov was supported by the scholarship of the President of the Russian Federation no. SP-3615.2018.1.

\section{References}

1. L.-L. Ke, Y.-S. Wang, Int. J. Solids Struct., 43, 5779 (2006)

2. T.-J. Liu, Y.-S. Wang, C. Zhang, F. Arch. Appl. Mech. 78, 267 (2008)

3. A.S. Vasiliev, M.V. Swain, S.M. Aizikovich, E.V. Sadyrin, Arch. Appl. Mech. 86, 1247 (2016)

4. Yu. Tokovyy, C.-C. Ma, J. Appl. Mech. 83, 111007 (2016).

5. I.I. Kudish, S.S. Volkov, A.S. Vasiliev, S.M. Aizikovich, J. Tribology, 138, doi:10.1115/1.4030956 (2016)

6. I.I. Kudish, S.S. Volkov, A.S. Vasiliev, S.M. Aizikovich, J. Tribology, 138, doi: 10.1115/1.4030958 (2016)

7. I.I. Kudish, S.S. Volkov, A.S. Vasiliev, S.M. Aizikovich, J. Tribology, 139, doi: 10.1115/1.4033360 (2017)

8. I.I. Kudish, S.S. Volkov, A.S. Vasiliev, S.M. Aizikovich, Math. Mech. Solids, doi: 10.1177/1081286517704689 (2017)

9. I.I. Kudish, S.S. Volkov, A.S. Vasiliev, S.M. Aizikovich, Math. Mech. Solids, doi: $10.1177 / 1081286517704690$ (2017)

10. I.I. Kudish, J. Tribology, 138, doi: 10.1115/1.4030788 (2016)

11. S.M. Aizikovich, A.S. Vasiliev, J. Appl. Math. Mech., 77 (2013)

12. S.M. Aizikovich, J. Appl. Math. Mech., 54 (1990) 